HIP-2009-29/TH

\title{
Inhomogeneous Structures in Holographic Superfluids: I. Dark Solitons
}

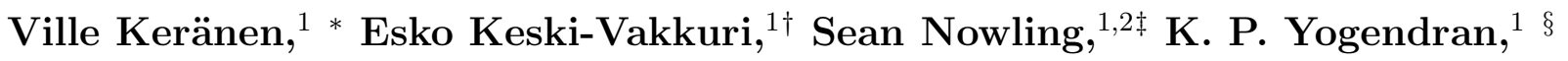 \\ ${ }^{1}$ Helsinki Institute of Physics \\ P.O.Box 64, FIN-00014 University of Helsinki, Finland \\ ${ }^{2}$ Department of Mathematics and Statistics \\ P.O.Box 68, FIN-00014 University of Helsinki, Finland
}

\begin{abstract}
We begin an investigation of inhomogeneous structures in holographic superfluids. As a first example, we study domain wall like defects in the $3+1$ dimensional Einstein-Maxwell-Higgs theory, which was developed as a dual model for a holographic superconductor. In [1], we reported on such "dark solitons" in holographic superfluids. In this work, we present an extensive numerical study of their properties, working in the probe limit. We construct dark solitons for two possible condensing operators, and find that both of them share common features with their standard superfluid counterparts. However, both are characterized by two distinct coherence length scales (one for order parameter, one for charge condensate). We study the relative charge depletion factor and find that solitons in the two different condensates have very distinct depletion characteristics. We also study quasiparticle excitations above the holographic superfluid, and find that the scale of the excitations is comparable to the soliton coherence length scales.
\end{abstract}

*ville.keranen@helsinki.fi

†esko.keski-vakkuri@helsinki.fi

‡sean.nowling@helsinki.fi

§yogendran.kalpat@helsinki.fi 


\section{Introduction}

The AdS/CFT correspondence [2-4] - or gauge-gravity duality, as its more phenomenological incarnation is called - is being used as a tool to study an increasing variety of strongly interacting systems. When the system is also "close" to being conformal, it is natural to try to construct a holographic gravity dual model. The main practical advantage of holography is that it allows a new, computationally simpler framework to explore quantum systems which are outside the reach of conventional perturbative techniques. The situation of interest in this document will be a system in which a $\mathrm{U}(1)$ symmetry is spontaneously broken, as is relevant for superfluidity and superconductivity.

Superfluidity may occur in a system at low temperatures when the ground state becomes occupied by a macroscopic number of particles. This is known to occur in both interacting bosonic systems (Bose-Einstein condensates, BEC) and interacting fermions with Bardeen-Cooper-Schrieffer pairing (BCS). In fact, cold atomic systems can display both BEC-like and BCS-like superfluidity and even a smooth transition between them.

It is not at all obvious what kinds of superfluids may be realized holographically. A prototype example is the recently constructed gravitational dual theory [6], building on the model [7], for the purpose or modeling BCS superconductors [6,8] or relativistic superfluids with a spontaneously broken global U(1) symmetry [9]. The model was also extended to an effective theory for some properties of a class of quantum Hall fluids [10]. Typically, in systems that are dual to AdS models, one finds both fermionic and bosonic excitations. Thus, one might hope to be able to holographically model both BEC-like and BCS-like superfluidity, as well as more complicated fluids with mixed behavior. For closer contact with real world cold atom systems, a required ingredient would also be manifest non-relativistic symmetry, for which the first holographic models were studied in $[11,12]$. In this paper, and in the associated sequence of works to follow, we will focus on studying extended configurations in the model [6], and we will find a richer variety of possibilities than previously expected.

Hydrodynamic properties of the relativistic holographic superfluid have been explored in $[13,14]$ - the latter in particular argues that the superfluid does not obey the Landau relationship between sound velocities. In [15], masses of quasiparticles were determined analytically near $T_{c}$ and substantially extended in [16] (to other values of the mass of the bulk scalar field as well).

Another basic fact is that conventional superfluids are known to support long lived spatially inhomogeneous configurations with nontrivial topology. An experimentally and theoretically interesting class of defects, found in superfluids, are domain wall like defects called dark solitons. A dark soliton is an interface of reduced (charge) density between two superfluid phases, with the order parameter changing sign across the interface. Holographic counterparts were recently reported by us in [1]. Apart 
from curiosity, there are several reasons to study such solitons, a primary one being to explore their properties at strong coupling, a difficult problem using traditional methods. Although the gravity dual has different symmetries and microscopic properties from the real life cold atomic systems, there may be some universal features. At the very least it provides a "spherical cow" example allowing one to study properties of a class of dark solitons at strong coupling.

The holographic dark solitons are also interesting solutions in their own right they turn out to reveal important information about the different ways of condensing operators in [6] and about the nature of the holographic superfluid. First of all, we find that the solitons have two distinct length scales: one for the charge density and one for the order parameter. This behavior is in contrast to a single scale found in simple solutions to the Gross-Pitaevskii equation for superfluids. Furthermore, by studying the degree of charge density depletion we discover that the two different choices for the condensing operator, discussed in [6], lead to different properties for the associated superfluids. We will also compare the length scales as set by these solitonic configurations with the microscopic masses of quasiparticles.

This document is organized as follows. We begin with a brief discussion of the holographic description of a superfluid. The configurations of interest are obtained by solving a system of partial differential equations in AdS space. These equations and the boundary conditions - which sustain the soliton - are presented in Section 2 . However, the equations seem intractable analytically. We therefore have to numerically solve the equations. The discretization of the equations and the method we use to solve the equations are presented in Section 3. In order to identify properties of interest to be obtained from the numerical solutions, we will briefly discuss the GrossPitaevskii equations in Section 4. We will then present our results for the holographic dark solitons and conclude with a discussion of the results and future directions.

\section{Holographic Description}

An explicit holographic modeling of a superfluid system was constructed in [6] following the ideas in [7] (closely related ideas also appeared in [17]). One considers a system consisting of a complex scalar field $\Psi$ interacting with a $\mathrm{U}(1)$ gauge field $F_{\mu \nu}=\partial_{\mu} A_{\nu}-\partial_{\nu} A_{\mu}$ in $3+1$ dimensions in the presence of gravity with a cosmological constant $\mathrm{E}$. The action for this system is

$$
\mathcal{S}=\int d^{3} x d z \sqrt{-g}\left[\frac{1}{2 \kappa_{4}^{2}}(R-12 \Lambda)+\frac{1}{q^{2}}\left(-\frac{1}{4} F_{\mu \nu} F^{\mu \nu}-m^{2} \Psi \bar{\Psi}-D_{\mu} \Psi D^{\mu} \bar{\Psi}\right)\right]
$$

where the covariant derivative $D_{\mu} \Psi=\left(\partial_{\mu}-i A_{\mu}\right) \Psi$ and $q$ may be identified with the charge of the scalar field 1 . Focusing on just these degrees of freedom (rather

\footnotetext{
${ }^{1}$ We have rescaled the fields such that $q$ only appears in front of the matter action.
} 
than keeping all the fields required for a UV complete gravity theory), amounts to concentrating on the most relevant operators of the field theory. One could include other fields (say, a fermion field which would be dual to fermionic quasiparticles). In keeping with the usual terminology, we will refer to the fields of the gravitational theory as the "bulk" fields in what follows.

The AdS/CFT correspondence [2] requires us to find solutions to the equations of motion of the $4 \mathrm{D}$-gravitating system, such that the metric is asymptotically AdS. The properties of the dual field theory are then "read off" from the asymptotic behavior of the various fields of this gravitating system ( $[3,4]$, see the review [5]).

In our case, since we are trying to study a spatially inhomogeneous configuration, solving the full gravitating system is a difficult problem. Therefore, we consider a limit in which the energy density in the complex scalar field and the Maxwell field (in 4D) is scaled to zero. This may be achieved by taking $\frac{\kappa_{4}}{q} \rightarrow 0$ keeping the field values $\Psi, A_{\mu}$ finite. In this limit (termed the probe limit in the AdS literature) the metric is determined, independent of the matter fields, to be a 4-D planar AdS black hole with a metric

$$
d s^{2}=L^{2}\left(-\frac{f d t^{2}}{z^{2}}+\frac{d z^{2}}{f z^{2}}+\frac{d \vec{x}^{2}}{z^{2}}\right), \quad f(z)=1-\left(\frac{z}{z_{T}}\right)^{3} .
$$

The Hawking temperature of the black hole, $T_{H}=\frac{3}{4 \pi z_{T}}$, is identified as the equilibrium temperature of the dual field theory.

The complex scalar field and gauge field then propagate on this background and their dynamics is determined by an action

$$
\mathcal{S}=\int d^{3} x d z \sqrt{-g}\left(-\frac{1}{4} F_{\mu \nu} F^{\mu \nu}-m^{2} \Psi \bar{\Psi}-D_{\mu} \Psi D^{\mu} \bar{\Psi}\right)
$$

where the covariant derivative $D_{\mu} \Psi=\left(\partial_{\mu}-i A_{\mu}\right) \Psi$ and the various indices are contracted using the metric given above.

If we rescale

$$
(z, x) \rightarrow z_{T}(z, x) \quad A \rightarrow \frac{A}{z_{T}} \quad \Psi \rightarrow \frac{\Psi}{z_{T}}
$$

then the new co-ordinates $z, x$ are dimensionless as are the new fields $\Psi, A$. Further, all dependence on $z_{T}$ is also removed.

The equations of motion for this system are

$$
\begin{aligned}
0 & =\frac{1}{\sqrt{-g}} D_{\mu}\left(\sqrt{-g} D^{\mu} \Psi\right)-m^{2} \Psi \\
0 & =\frac{1}{\sqrt{-g}} \partial_{\mu}\left(\sqrt{-g} F^{\mu \nu}\right)+i\left(\Psi \partial^{\nu} \bar{\Psi}-\bar{\Psi} \partial^{\nu} \Psi\right)+2 A^{\nu} \Psi \bar{\Psi}
\end{aligned}
$$

In this work we will primarily be interested in studying static kink solutions in the field theory and therefore the fields only have dependence on $z$ and one spatial variable, 
$x$. In the gauge where $A_{z}=0$, the $A_{z}$ equation is satisfied if $\Psi$ is taken to be real and $A_{x}=0$. We will assume translation invariance in the $y$-direction and set $A_{y}=0$. Then the only nonzero component of the gauge field is $A_{0}=A(z, x)$

It is important to note that, after gauge fixing, there is a residual $\mathbb{Z}_{2}$ symmetry under which the scalar field changes sign. When studying dark soliton solutions to the equations of motion, the scalar field changes sign as a function of $x, \lim _{x \rightarrow \pm \infty} \Psi(x)=$ $\pm|\Psi(\infty)|$. One might think that this sign flip may also be gauged away, but this would require a gauge transformation that is singular as the scalar field crosses through zero, and hence is not an allowed gauge transformation.

It is convenient to redefine the field slightly, $\Psi=z \tilde{R} / \sqrt{2}$, and rewrite equations of motion

$$
\begin{aligned}
f \tilde{R}^{\prime \prime}+f^{\prime} \tilde{R}^{\prime}-z \tilde{R}+\partial_{x}^{2} \tilde{R}+\tilde{R}\left(\frac{A^{2}}{f}\right) & =0 \\
f A^{\prime \prime}+\partial_{x}^{2} A-\tilde{R}^{2} A & =0
\end{aligned}
$$

\subsection{Bulk-boundary dictionary}

Typically, in AdS spacetimes, the solutions to the equations of motion may be segregated into "normalizable" and "non-normalizable" parts determined by their leading $z$ behavior as one approaches the boundary. According to the AdS/CFT dictionary $[3-5]$ the two have different interpretations in the dual field theory. The boundary values of the non-normalizable modes are interpreted as sources for the operators of the dual field theory. The normalizable modes are then identified with the vacuum expectation values of the corresponding operators sourced by the non-normalizable modes [18]. The scaling behavior of the dual operator is also fixed and may be extracted from the asymptotic behavior of the normalizable modes as functions of $z$. This is explicitly realized by identifying the generating functional of connected correlation functions, $W[J]$, of the strongly coupled field theory with the on-shell action for the bulk gravity theory (the bulk fields have boundary values $\phi_{\partial}=J .([3,4])$

$$
\left.S_{\text {Grav., o.s. }}\right|_{\phi_{\partial}=J}=W_{Q F T}[J] .
$$

One simple way to see the relationship between normalizable modes and expectation values is to consider the change in the action of the gravity theory caused by infinitesimal changes in the "non-normalizable" gravity modes (field theory sources). Upon partial integration and the inclusion of a suitable counterterm, the variation of the action (11) in terms of the fields $\Psi, A$, becomes

$$
\begin{aligned}
\delta S= & -\int d^{4} x \partial_{\mu}\left(\delta \Psi \sqrt{g} \partial^{\mu} \Psi\right)+\frac{1}{2} \partial_{\mu}\left(\sqrt{g} \delta A F^{\mu 0}\right) \\
& +\int d^{4} x \delta \Psi \mathcal{E}(\Psi)+\frac{1}{2} \delta A_{\nu} \mathcal{E}(A) \\
& + \text { terms higher order in the variations. }
\end{aligned}
$$


We have grouped the equations of motions in (5) as $\mathcal{E}(\Psi)$ and $\mathcal{E}(A)$ for brevity. Rewriting the surface terms as in the AdS prescription gives us

$$
\begin{aligned}
\delta S= & -\left.\int d^{3} x\left[\left(\delta \Psi \sqrt{g} \partial^{z} \Psi\right)+\left(\sqrt{g} \delta A F^{z 0}\right)\right]\right|_{z=0} \\
& +\int d^{4} x \delta \Psi \mathcal{E}(\Psi)+\frac{1}{2} \delta A \mathcal{E}(A) \\
& + \text { terms higher order in the variations. }
\end{aligned}
$$

contributions only from the first line in the above.

As in [6], we shall take the scalar field to satisfy $m^{2}=-2 / L^{2}$. For this mass value, there are two quantizations for scalar fields in any asymptotically $\mathrm{AdS}_{4}$ spacetime [19]. The two quantizations correspond to exchanging the role of source and expectation value in the dual field theory.

More explicitly, it can be easily seen that, close to the boundary at $z=0$, solutions to the equations of motion must behave as

$$
\tilde{R} \sim \tilde{R}^{(1)}+z \tilde{R}^{(2)}+\ldots, \quad A \sim A^{(0)}+z A^{(1)}+\ldots
$$

in an expansion of the Frobenius type along the $\mathrm{z}$-direction. We can regard $\tilde{R}^{(1)}$ as the source for a charged, dimension 2 operator

$$
\tilde{R}^{(1)}=z_{T} J^{(2)} \quad \tilde{R}^{(2)}=z_{T}^{2}\left\langle\mathcal{O}_{2}\right\rangle \quad A_{0}^{(0)}=z_{T} \mu \quad A_{0}^{(1)}=z_{T}^{2} \rho .
$$

where $\left\langle\mathrm{O}_{2}\right\rangle$ is the charged operator, $\mu$ is the chemical potential, and $\rho$ is the charge density (all in the dual field theory). One could motivate the identification of the asymptotic value $A_{0}^{(0)}=z_{T} \mu$ by noting that, in Euclidean space, a vev of $A_{0}$ is naturally interpreted as a chemical potential (since it minimally couples to a conserved charge).

In the second quantization scheme, we identify $\tilde{R}^{(2)}$ as the source for a charged, dimension 1 operator

$$
\tilde{R}^{(1)}=z_{T}\left\langle\mathcal{O}_{1}\right\rangle \quad \tilde{R}^{(2)}=z_{T}^{2} J^{(1)} \quad A_{\mu}^{(0)}=z_{T} \mu \quad A_{\mu}^{(1)}=z_{T}^{2} \rho .
$$

(we will use $\psi$ to denote the condensate in what follows). The various prefactors come from the rescaling of $z$ by $z_{T}$ so that the fields in the bulk are dimensionless. Solutions to the gravitational equations of motion with nonzero non-normalizable components are interpreted in the dual field theory as deforming the Hamiltonian, $\delta \mathcal{H} \sim \int d^{3} x J^{(i)} \mathcal{O}^{(i)}$ and would hence change the ground state of the theory.

To obtain a unique solution to the bulk equations of motion, we need to impose boundary and regularity conditions. As in [6], we want to study the spontaneous breaking of the $U(1)$ symmetry unperturbed by any sources, so we will impose boundary conditions such that in each quantization the source terms are turned off. 
In addition, we impose the following regularity conditions at the horizon (we assume that $R$ is regular at the horizon)

$$
f^{\prime} \tilde{R}^{\prime}-z \tilde{R}+\partial_{x}^{2} \tilde{R}=0 \quad A_{0}(z=1, x)=0
$$

(the condition on the gauge field has been argued to be a regularity condition [7]). Because the differential equations are elliptic (7) outside the horizon, Dirichlet/Neumann boundary conditions along the boundary (at $z=0$ ) and regularity (at the horizon) are sufficient to fix a unique solution (a useful analogy is with Poisson equations).

\subsection{Review of earlier results}

One of the features of the AdS/CFT correspondence is that global symmetries of the field theory system appear gauged in the gravitational theory. Given this, the $U(1)$ gauge symmetry in the gravitational Lagrangian (1) is interpreted as corresponding to a global $U(1)$ symmetry of the dual field theory system. And hence, the bulk charged scalar field $\Psi$ maps into a field theory operator $\mathcal{O}_{i}$ that is "charged" under the global $U(1)$. We can now study this system to see if it has ground states in which these charged operators pick up nonzero vacuum expectation values (vevs) in the limit where all external sources for charged operators are turned off.

In the works of [6], it was shown that for $\mu \geq \mu_{c}$ (or equivalently small $T$, since there is only one independent parameter $\tilde{\mu}=\frac{3 \mu}{4 \pi T}$ ), one can find nontrivial solutions for the scalar field equations displaying spontaneous symmetry breaking in the dual field theory. Thus, for low enough temperature (or large enough $\mu$ ) we obtain superfluidity (strictly, we have only argued for a condensate, that this is indeed a superfluid has also been established by showing the existence of a hydrodynamic mode [9]). The critical value of $\mu$ (or $T$ ) depends on whether we consider $\tilde{R}^{(1)}$ or $\tilde{R}^{(2)}$ as our normalizable mode (or equivalently, as the vev of the order parameter).

The graph of typical solutions look as in Fig. 1 wherein we plot the two solutions obtained by condensing either operator $\mathcal{O}_{1}$ (dotted line) or $\mathcal{O}_{2}$ (dashed line) We also
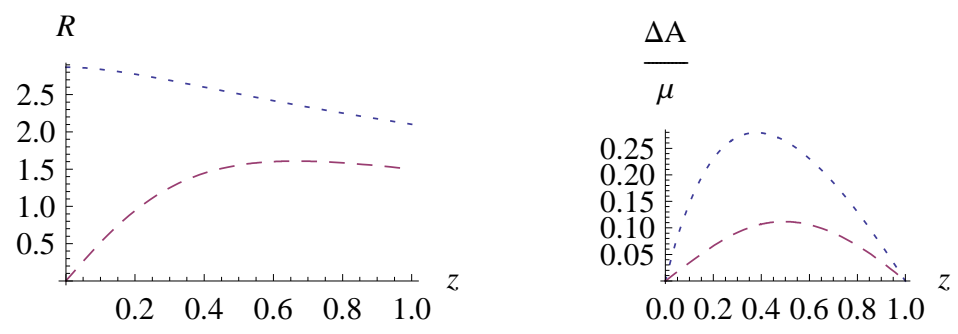

Figure 1: Scalar field and Gauge field profile: The dotted line refers to $\mathcal{O}_{1}$ and the dashed line to $\mathcal{O}_{2}$ type condensate

note that at any temperature (or chemical potential) we have a solution where the 
condensate is absent $A(z)=\tilde{\mu}(1-z)$ and $\Psi=0$. Such a solution is energetically disfavored at low temperatures $\left(T<T_{c}\right.$ or $\left.\mu>\mu_{c}\right)$. [9]

The features in the bulk gauge field are highlighted better if we graph a subtracted potential $\Delta A(z)=A(z) / A(0)-(1-z)$ which may be interpreted as charge density over and above the zero condensate value. It is seen in Fig. 1 that the bulk gauge field profile is quite similar in the two cases. One feature of potential interest is that the presence of the condensate changes the charge density by little (vide the range of the vertical axis), at least for small chemical potentials.

We also plot the charge density and condensate as a function of the holographic direction $z$. One of the interesting differences between the two types of solutions is that when $\mathcal{O}_{2}$ condenses, the condensate always vanishes at a point $z=z_{c}$. Beyond this $z_{c}$ value the condensates is always monotonically decreasing as one approaches the horizon. For $\mathcal{O}_{1}$ condensates, the condensate is monotonic as a function of $z$. The Landau-Ginzburg approach suggests that the square of the condensate should be
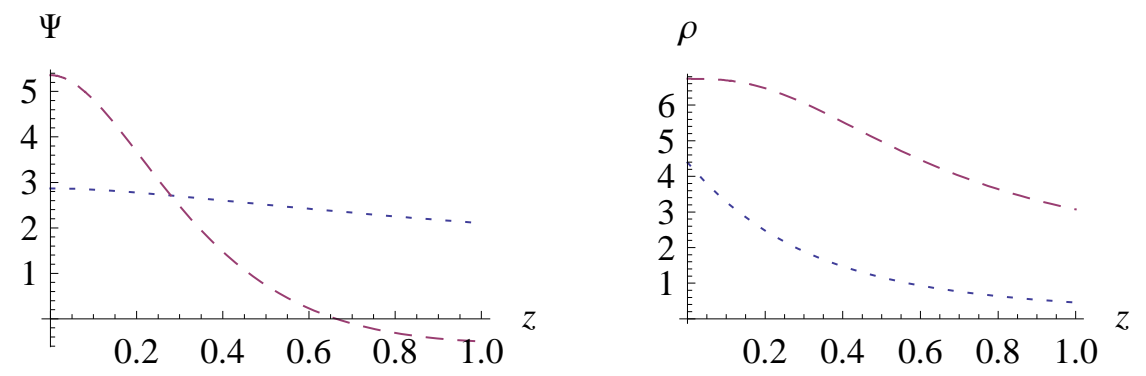

Figure 2: The condensate and charge density profile: The dotted line refers to $\mathcal{O}_{1}$ and the dashed line to $\mathcal{O}_{2}$ type condensate

function of $\left(\mu-\mu_{c}\right)$ (26). As can be seen from Fig. 3, for small values of $\frac{\left(\mu-\mu_{c}\right)}{\mu_{c}}$, the curve may be approximated by a straight line. However, for somewhat larger values, the slope changes - and the fitting functions require higher powers.

The equation of state for this system - namely the graph of the charge density as a function of the chemical potential for fixed temperature looks as in the Fig. 4. Close to the phase transition, the charge density and chemical potential are linearly related, but near $T=0$ (large $\mu$ ), this is no longer true.

One could expect that the condensate should be a linear function of the charge density - if one imagines that the microscopic system consists of fermions, then both the charge density and the condensate are proportional to the vev of $\psi \psi$ - where $\psi$ is the fermion many body wavefunction. The condensate is indeed a linear function of the charge density at large values of the chemical potential (i.e., close to $T=0$ ) but near the phase transition it seems that increasing the condensate does not increase the charge density by a considerable amount. 


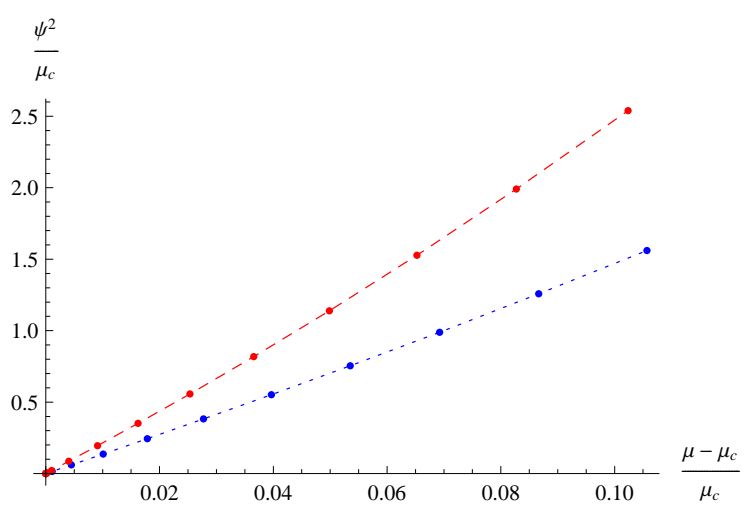

Figure 3: The condensate as a function of the chemical potential: Upper curve is $\mathcal{O}_{2}$, lower $\mathcal{O}_{1}$.
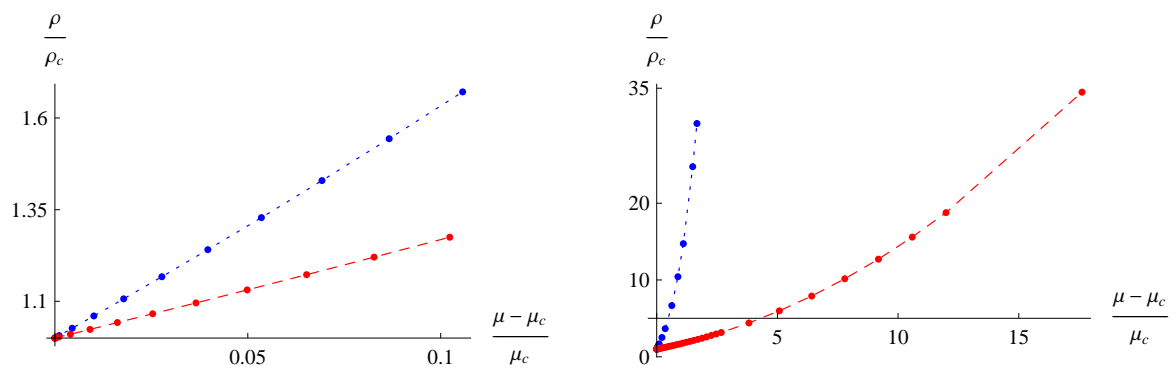

Figure 4: The charge density vs. chemical potential: Upper curve is $\mathcal{O}_{2}$, lower $\mathcal{O}_{1}$.

\section{Numerical Methods for Dark solitons}

The bulk equations of motion (17) seem to be intractable even in the homogeneous case. Therefore, we resort to numerical methods to solve them. Since we are interested in inhomogeneous solutions, shooting methods become unwieldy (in this regard, see [33] for a tour de force of numerical work in a closely related context). Therefore, because the differential equations are elliptic outside the horizon, it is natural to use relaxation methods to find solutions.

More precisely, to simulate the differential equations, we place the system in a large box of size $1 \times L_{x}$ in the $z$ and $x$ directions respectively. We discretize this box using a lattice, and then solve a discretized version of the differential equation on the lattice using a Gauss-Seidel routine. The Gauss-Seidel strategy is to start with a seed configuration which obeys appropriate boundary conditions. We can then use a discrete representation of the differential equations to "relax" this configuration towards a solution. If the lattice is sufficiently fine, the elliptic nature of the problem implies that the seed configuration will flow to an exact solution to the differential equation eventually. 


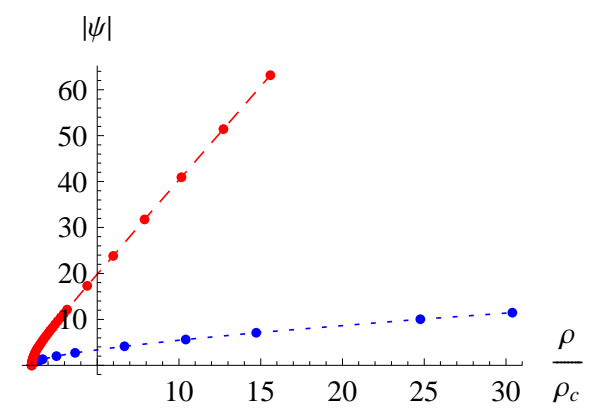

Figure 5: The condensate as a function of the charge density (upper curve is $\mathcal{O}_{1}$ condensate)

We set our conventions by labeling the $z$ and $x$ positions with $i \in[0, M]$ and $j \in[0, N]$ respectively and use step sizes $h_{z}$ and $h_{x}$. We will denote the lattice fields as $(\tilde{R}, A) \rightarrow\left(\tilde{R}_{i, j}, A_{i, j}\right)$.

The discretization of these equations (77) has to be done separately for the interior of the lattice and the edges to at least second order in the lattice spacings. In the interior, when $i \neq 1, M j \neq 1, N$, we can use the center difference formulae for the derivatives,

$$
\begin{aligned}
\left.\frac{\partial f(x)}{\partial x}\right|_{x=x_{i}} & =\frac{f\left(x_{i+1}\right)-f\left(x_{i-1}\right)}{2 h_{x}}+\mathcal{O}\left(h_{x}^{2}\right) \\
\left.\frac{\partial^{2} f(x)}{\partial x^{2}}\right|_{x=x_{i}} & =\frac{f\left(x_{i+1}\right)-2 f\left(x_{i}\right)+f\left(x_{i-1}\right)}{h_{x}^{2}}+\mathcal{O}\left(h_{x}^{2}\right)
\end{aligned}
$$

and therefore we get the following algebraic equations

$$
\begin{aligned}
\tilde{R}_{00} & =\frac{\left(\left(\frac{f}{h_{z}^{2}}+\frac{f^{\prime}}{2 h_{z}}\right) \tilde{R}_{+0}+\left(\frac{f}{h_{z}^{2}}-\frac{f^{\prime}}{2 h_{z}}\right) \tilde{R}_{-0}+\frac{\tilde{R}_{0+}+\tilde{R}_{0-}}{h_{x}^{2}}\right)}{\left(z-\frac{A_{00}^{2}}{f}+\frac{2}{h_{x}^{2}}+\frac{2 f}{h_{z}^{2}}\right)} \\
A_{00} & =\frac{\frac{f}{h_{z}^{2}}\left(A_{+0}+A_{-0}\right)+\frac{1}{h_{x}^{2}}\left(A_{0+}+A_{0-}\right)}{\left(\tilde{R}_{00}^{2}+\frac{2}{h_{x}^{2}}+\frac{2 f}{h_{z}^{2}}\right)}
\end{aligned}
$$

The above equations are to be understood as follows. At a site $i, j$, the value of the scalar field $R_{i, j}$ which we label as $R_{00}$ is determined in terms of its neighbors $R_{i \pm 1, j}=R_{ \pm 0}$ etc by the above equations. In addition, $z$ refers to the lattice value $z_{i}$ and $f$ refers to $f\left(z_{i}\right)$ and $f^{\prime}$ must be replaced by the center difference formula at the lattice site.

At the spatial edges (in the bulk) when $x= \pm L_{x} / 2$ and $z \neq 0,1$, we simply use one-sided representations of the finite difference derivatives and again impose the equations of motion. We have also checked that one could also employ Neumann boundary conditions at $x= \pm \frac{L}{2}$ without changing the numerically determined solutions, as long as $L$ is sufficiently large. 
At the horizon, we impose regularity conditions (15) appropriately discretized. At the boundary of AdS space, we have to numerically impose the boundary conditions

$$
\begin{array}{ll}
\mathcal{O}_{1} \text { case }: A(z=0, x)=\mu=\text { constant, } & \frac{\partial \tilde{R}}{\partial z}(z=0, x)=0 \\
\mathcal{O}_{2} \text { case }: A(z=0, x)=\mu=\text { constant, } & \tilde{R}(z=0, x)=0 .
\end{array}
$$

By cycling through the lattice, imposing either the equations of motion, regularity, or boundary conditions depending upon the position, an initial seed configuration relaxes to a solution to the algebraic equations satisfying the appropriate boundary conditions.

As a first check of our algorithm, we checked that we can obtain the symmetry breaking solutions obtained by [6] that correspond to having a homogeneous phase without any dark solitons. For the $\mathcal{O}_{2}$ condensate for instance, we set the chemical potential at the boundary to a fixed value, and $\tilde{R}(0, x)=0$, and allow fairly arbitrary seed field values in the bulk of the lattice (as well as at the horizon). Upon subsequent iteration, we obtain a translationally invariant solutions that match the numerical solutions obtained by solving the corresponding one dimensional problem using Mathematica's NDSolve to remarkable accuracy even on fairly modest lattices. For the $\mathcal{O}_{1}$ condensate we enforced $\partial_{z} \tilde{R}(0, x)=0$ instead. Again, after iterating one finds spatially homogeneous solutions which agree with [6].

\subsection{Constructing Dark Solitons}

The dark solitons were constructed, numerically, as follows. We first chose a seed configuration that asymptotes to solutions in [6] far away from the interface. The initial configuration is assumed to be odd in the x-direction. We then numerically iterate the seed configurations until it relaxes to a stable configuration (we do this on several lattice sizes for each soliton). In actual calculations, we are forced to use a cutoff at a distance $\epsilon=10^{-10}$ from the horizon at $z=1$. We have checked that this value of $\varepsilon$ does not affect the results quoted.

A typical solution so obtained is shown in Fig. 6] for the $\mathcal{O}_{1}$ case. In the gauge field plot, we have subtracted a linear part $\mu(1-z)$. Since this is an $\mathcal{O}_{1}$ condensate, the boundary values of the scalar field are non-vanishing. A corresponding typical solution for the $\mathcal{O}_{2}$ case is shown in Fig. 7, In this case the boundary value of the scalar field is zero and the derivative of the scalar field, $\partial_{Z} \tilde{R}$, is identified with the condensate. magnitude of

Further, starting with one such solution, we can perturb the solution by an arbitrary deformation that preserves the boundary values. We have seen that the perturbed configuration relaxes back to the original starting solution rapidly (in iteration time). This is an indirect argument for the stability of the solution as well. 

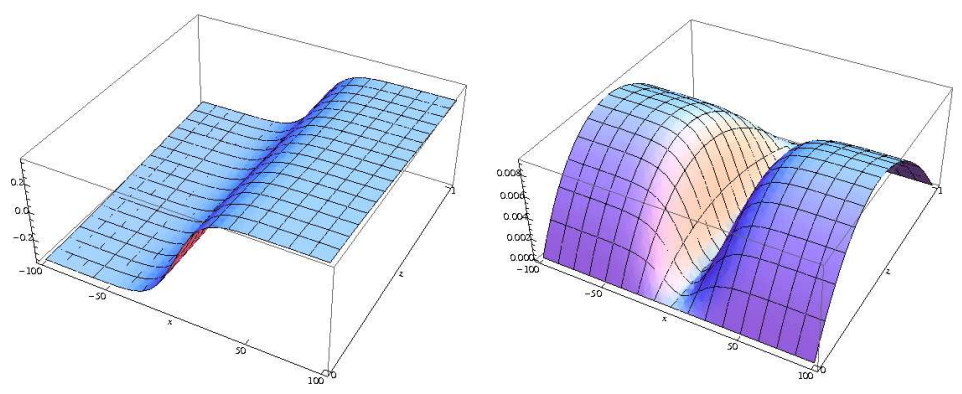

Figure 6: $\mathcal{O}_{1}$ case: The bulk solution (Scalar field on the left and subtracted gauge field on the right).
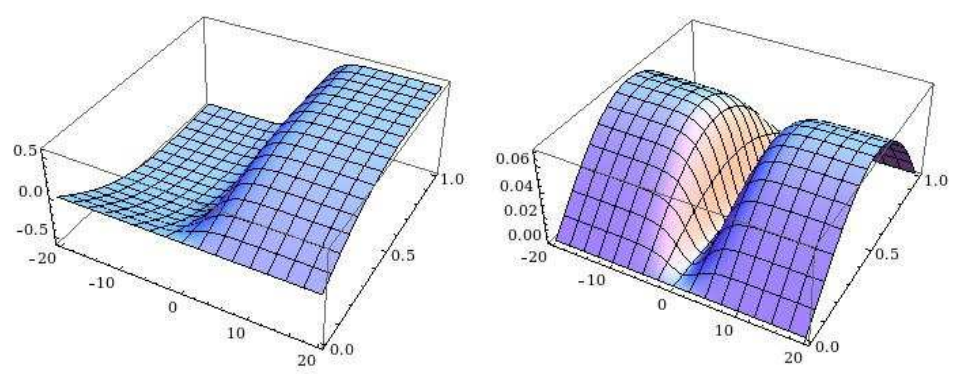

Figure 7: $\mathcal{O}_{2}$ case: The bulk solution (Scalar field on the left and subtracted gauge field on the right).

\subsection{Numerical Errors}

There are two main sources of errors in the relaxation method described above. First, there is the discretization error caused by using a lattice description. This may be countered in two obvious ways. We could use discrete representations of derivatives that are of higher order in $h_{x}$ and $h_{z}$ and/or use a finer lattice. We however resort to a version of the multigrid method. That is to say, we first solve the numerical system on a coarse lattice and then use this approximate solution to seed the starting configuration on a finer lattice. This procedure converges much faster than naively solving on the larger lattice. The second source of error is the degree to which we solve the algebraic equations obtained after discretizing the differential equations. The most obvious way to minimize the algebraic error is to simply let the system relax over more iterations.

As a measure of the error in our numerical solutions, we define a quantity which 
we call equation error, $\mathcal{E}$. This is the extent to which the numerically determined solutions do not solve the equations of motion schematically written as

$$
\text { E.O.M }\left(\tilde{R}_{\text {num }}, A_{\text {num }}\right)=\mathcal{E} .
$$

To evaluate $\mathcal{E}$, we can again discretize the equations of motion by now using n-th order representations for the various derivatives and evaluate $\mathcal{E}_{\text {num }}(n)=E \cdot O \cdot M_{n}\left(\tilde{R}_{\text {num }}, A_{\text {num }}\right)$. For a given numerical solution, we find that $\mathcal{E}_{\text {num }}(n)$ hardly changes for $n>5$. We may therefore use this numerically determined equation error (at large derivative ordern $>5$ ) as an approximation of the exact error in the numerical solution

$$
\left.\mathcal{E} \sim \mathcal{E}_{\text {num }}(n)\right|_{\text {large } n}
$$

In order to reduce the discretization error we may pass numerically obtained solutions obtained on coarse lattices on to finer lattices and repeat the process. In this manner it is possible to systematically reduce the equation error, $\mathcal{E}$. In principle this process could be continued indefinitely, although in reality we are limited to lattices of size less than $513 \times 513$ sites when using Mathematica on a desktop computer.

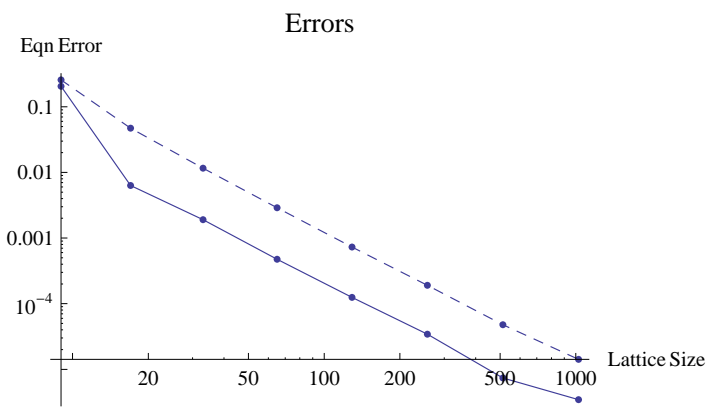

Figure 8: $\mathcal{E}_{\text {dis }}(7)$ as a function of lattice size. The dashed line is the maximum value of the $\tilde{R}$ equation of motion evaluated on our numerical solution and the solid line is the corresponding value for the gauge field

\subsubsection{Uncertainty Analysis}

Although we have introduced a measure of numerical error in the solutions, it would be more insightful to have a measure of the error in physical quantities. One way to do this comes from (11) which establishes the relationship between normalizable modes and operator expectation values. Specifically, the error in the condensate and charge density may be estimated by noting that since the equations of motion do not 
actually evaluate to zero,

$$
\begin{aligned}
\Delta\left(\left\langle O_{2}\right\rangle\right) & \sim \frac{1}{z_{T}^{2}} \int d^{4} x\left|\frac{\delta \tilde{R}}{\delta \tilde{R}^{(1)}}\right|\left|\mathcal{E}_{\text {num }}(\tilde{R})\right|+\frac{1}{2}\left|\frac{\delta A}{\delta \tilde{R}^{(1)}}\right|\left|\mathcal{E}_{\text {num }}(A)\right| \\
\Delta(\langle\rho\rangle) & \sim \int \frac{d^{4} x}{f(z)}\left|\frac{\delta \tilde{R}}{\delta A^{(0)}}\right|\left|\mathcal{E}_{\text {num }}(\tilde{R})\right|+\frac{1}{2}\left|\frac{\delta A}{\delta A^{(0)}}\right|\left|\mathcal{E}_{\text {num }}(A)\right|
\end{aligned}
$$

for the $\mathcal{O}_{2}$ condensate. Similar expressions may be obtained for the $\mathcal{O}_{1}$ condensate.

As a final input we need to evaluate the functional derivative of bulk fields with respect to their boundary values. We will approximate the functional derivatives by their values for the homogeneous solutions (as determined numerically using Mathematica's NDSolve routine). That is to say, we determine the change $\delta \tilde{R}(z)$ when we change the boundary values by $\delta \tilde{R}^{(1)}$. We have checked that this latter approximation is a good estimate of the actual functional derivative $\frac{\delta \tilde{R}}{\delta \tilde{R}^{(1)}}$ even if we use the full inhomogeneous equations.

Such as they are, these error estimates do not represent upper bounds on the total error on spatially inhomogeneous solutions, but we do believe that they are a descriptive of the error in our numerical solutions.

\section{Dark Solitons}

In order to identify quantities of interest to be extracted from our numerical solutions, we will attempt to compare our holographic solitons with the soliton solutions of the Gross-Pitaevskii(GP) equation (the latter gives a coarse-grained description of a superfluid valid at long wavelengths). However, we emphasize that we will only be using the GP equation as a guide - the results indicate that the holographic solitons are not the same as the solution of the GP equation. We also note that the description of a superfluid system in terms of the GP equation alone is inadequate to capture the effects of significant density depletion in the superfluid (perhaps due to strong interaction effects) and also the effects of having a significant non-condensate fraction (at say high temperatures). In our results, we find that both these contributions seem to be present and significant in the holographic superfluids.

The order parameter of superfluidity is a complex scalar field (also called the condensate wave function)- for which one can write down equation of motion called the Gross-Pitaevskii equation

$$
-\frac{1}{2 m_{B}} \partial^{2} \psi+(V-\mu) \psi+g \psi|\psi|^{2}=0
$$

(we have dropped the time dependence in the above since we are only interested in static phenomena in this work). 
The dark soliton, in this language is a spatially varying solution of the GP equation which interpolates between the potential minima

$$
\psi(x \rightarrow \infty)=\Delta \quad \psi(x \rightarrow-\infty)=-\Delta
$$

If we further assume translational invariance in the y-direction, the GP equations (24) has a well known exact solution

$$
\psi=\sqrt{\frac{V-\mu}{g}} \tanh (x / \xi),
$$

which interpolates between the two minima at $\psi= \pm \Delta=\sqrt{\frac{V-\mu}{g}}$. The correlation length (or the healing length) $\xi$, can then be written in a useful form in terms of the parameters of the GP equation as

$$
\xi^{2}=\frac{1}{2 g \Delta^{2}}=\frac{1}{(V-\mu) m_{B}} .
$$

Although the GP equation is really relevant for a nonrelativistic system, it will be interesting to test these dependences of the coherence on the magnitude of the condensate and on the chemical potential. Finally, because the charge density is simply related to the order parameter $\rho \sim|\psi|^{2}$, the solution (26) has vanishing charge density at its core.

\subsection{Holographic results}
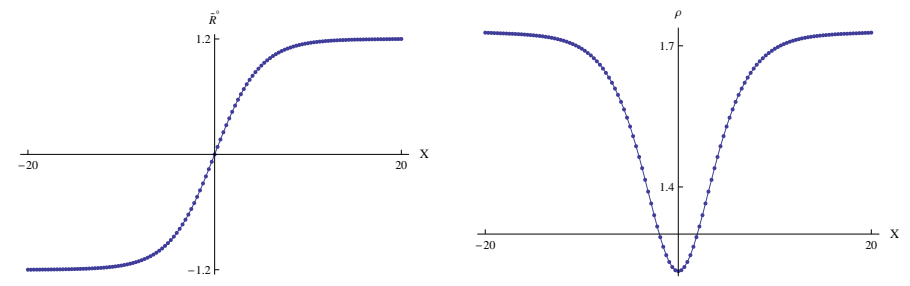

Figure 9: $\mathcal{O}_{1}$ Condensate and charge density as a function of $x$

From the numerical solutions (6,7) obtained by solving the bulk equations - we can extract the boundary profiles of the charge density $A^{(1)}$ and the condensate $R^{(1,2)}$ respectively. The plots as well as expectations from the GP equation suggest that the condensate can be fitted by a $\tanh \left(\frac{x}{\xi}\right)$ profile and hence plausibly, the charge density can be fitted by a $\operatorname{sech}^{2}\left(\frac{x}{\xi_{q}}\right)$ profile. These data points along with the best fit curve are shown in Fig. 9 for a typical $\mathcal{O}_{1}$ type of condensate. The results of a similar analysis for the $\mathcal{O}_{2}$ system is shown in Fig. 10. The profiles of the boundary observables are 

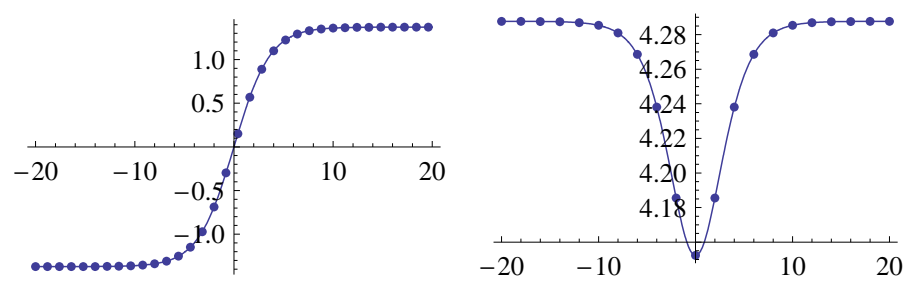

Figure 10: $\mathcal{O}_{2}$ Condensate and charge density as a function of $x$.

equally well fit for both $\mathcal{O}_{1}$ and $\mathcal{O}_{2}$ cases. Using a least square fit, we then extract the coherence length $\xi$ from the condensate profile and $\xi_{q}$ from the charge density. It must be noted here that the fit value of $\xi$ and $\xi_{q}$ are quite sensitive to the accuracy of the solutions. We have ensured that the equations are solved to an accuracy of $10^{-4}$ - that is to say, the maximum absolute value of the right hand side of the differential equation is less that $10^{-4}$.

Following our earlier discussion of the GP-equation (27), we plot the behaviour of the coherence length as a function of the condensate in Fig. [11. One of the first surprises is that $\xi$ is a linear function of the inverse condensate over a large range of chemical potential, although there is no obvious reason for it in this context.

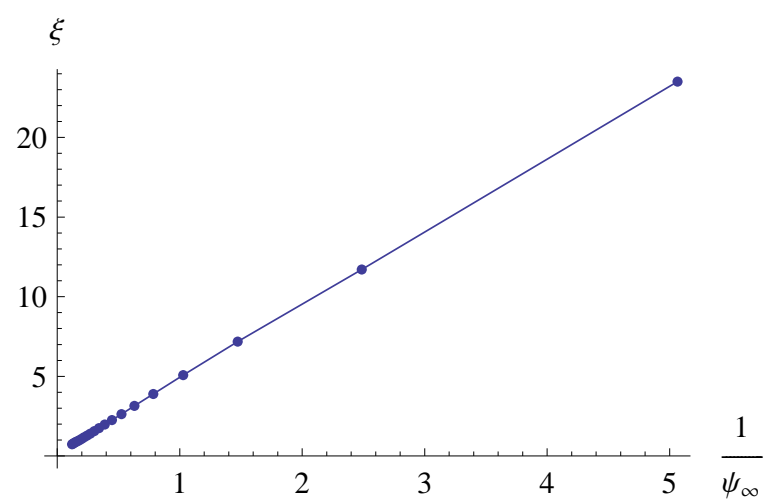

Figure 11: $\mathcal{O}_{2}$ Coherence length versus inverse condensate.

\subsection{Two Length Scales}

Secondly, again motivated by (27), we would like to determine the dependence of the coherence lengths $\xi$ on the chemical potential $\mu$. This is shown in Fig. 12, It is seen that for values of the chemical potential close to the critical value (i.e., $\mathrm{T}$ close to $T_{c}$ ), these mass scales are linear functions of the chemical potential (in this manuscript, we use the term mass scale interchangeably with inverse length scale). This is true for 

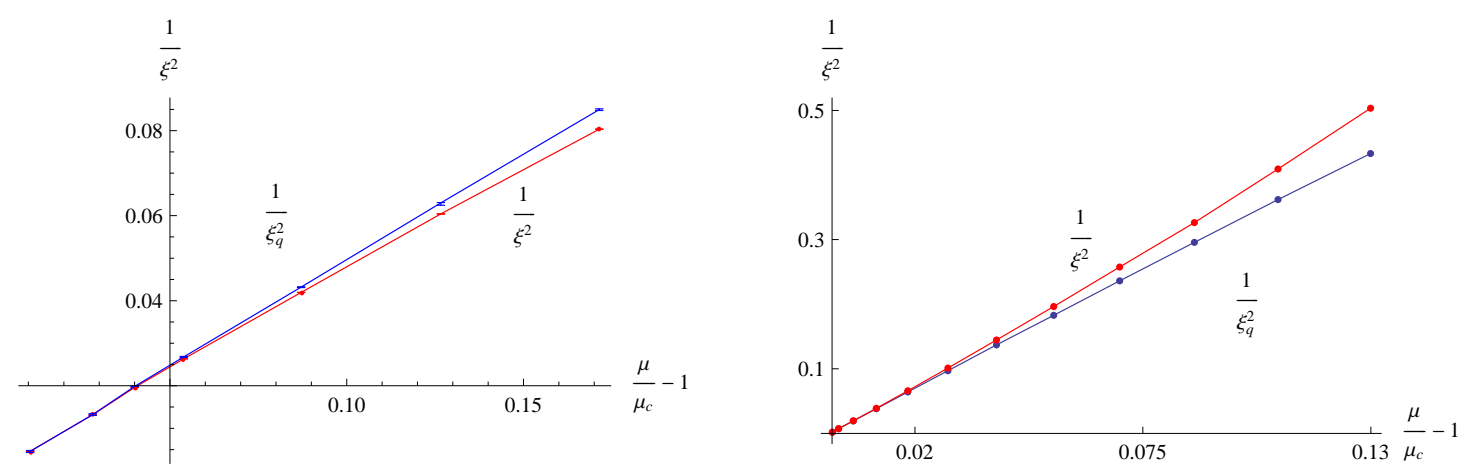

Figure 12: The coherence lengths as a function of the chemical potential $\left(\mathcal{O}_{1}\right.$ on the left and $\mathcal{O}_{2}$ on the right)

both the $\mathcal{O}_{1}$ and $\mathcal{O}_{2}$ solutions - but $\xi$ and $\xi_{q}$ have slightly different slopes as functions of the chemical potential.

This is in sharp contrast to what one might have expected from either GP-equation or even the bulk equations. The numerical solutions display different length scales for the condensate and charge density (for $\mu>\mu_{c}$ ). We have confirmed that this is not a numerical artefact in the following ways. As a first check, we plot the behavior of the two length scales as we increase lattice size in Fig. 13. We see that the

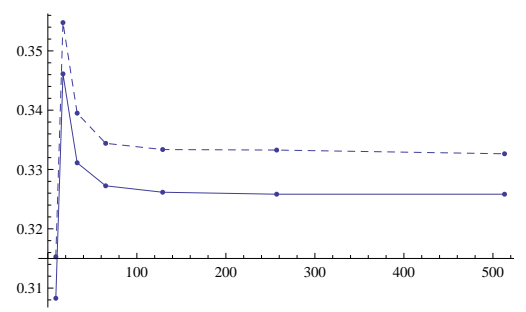

Figure 13: The best fit valued for $\xi$ (bottom) and $\xi_{q}$ (top) vs. lattice size for an $\mathcal{O}_{2}$ condensate at $\frac{\mu}{\mu_{c}}=1.03$.

difference between the $\xi$ 's saturates for large lattice sizes, indicating that errors due to discretization will not swamp the difference between the two scales.

We also plot the relative difference between $\xi$ and $\xi_{q}$ as a function of the chemical potential in Fig. 14. Following the earlier discussion on error estimate for the condensate and charge density profiles, we have determined error bars on these numbers (which have also been indicated in Fig. 14). Since the error bars do not overlap with zero (except close to $T_{c}$ ), it is clear that the difference in these length scales is not a numerical artefact. A sharp difference between the two kinds of condensates is seen in the observation that the length scale difference changes sign. 

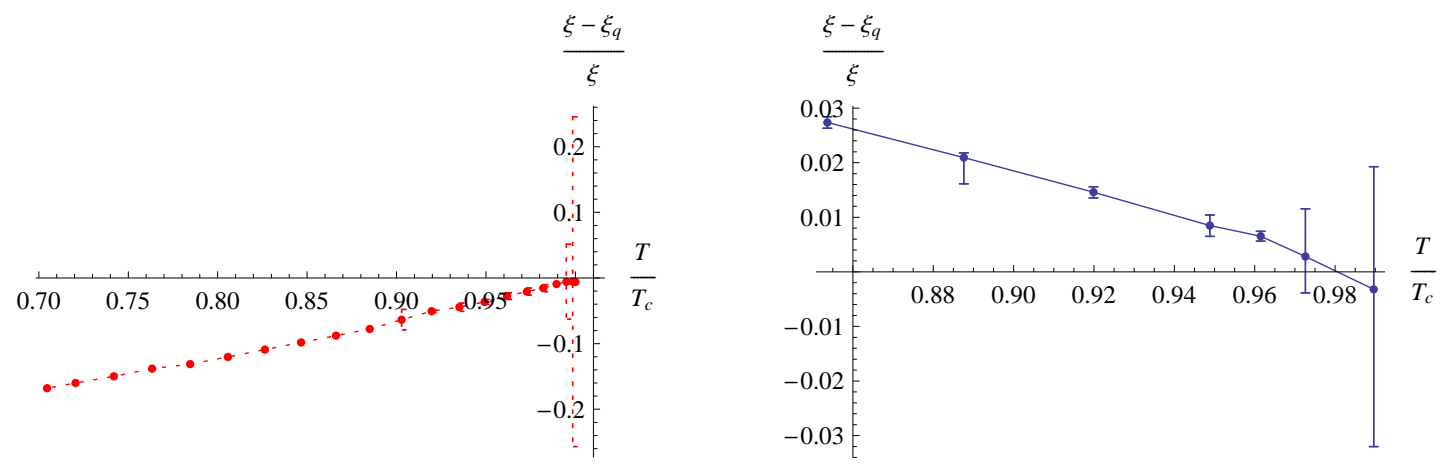

Figure 14: $\frac{\xi-\xi_{q}}{\xi}$ vs. $\frac{T}{T_{c}}$ : On the left is $\mathcal{O}_{2}$ and the right is $\mathcal{O}_{1}$

The existence of two distinct length scales leads one to expect an interesting layering effect as one nears the soliton's core. Two length scales would indicate that the relative fraction of charge density in the condensate versus non-condensate degrees of freedom varies as a function of $x$. For $\mathcal{O}_{1}$ we find that the condensate's length scale is larger than that of the total charge density. As one approaches the soliton from infinity, the $\mathcal{O}_{1}$ condensate starts to drops off before the total charge density, therefore the non-condensate contribution to the total charge density must be must be relatively over dense when compared to its asymptotic value. For $\mathcal{O}_{2}$, the relative order of the length scales is reversed, implying that the density in the non-condensate fraction must be relatively under dense near the dark soliton.

\subsection{Depletion fraction}

Another quantity of interest in these objects is the amount of density depletion at the core of the soliton. This is illustrated in the left panel of Fig. 15 where we plot the fractional density as a function of the distance from the core of the soliton (for $\frac{\mu}{\mu_{c}}=1.9$ ). One sees that there is a striking difference in the amount of density depletion at the core between $\mathcal{O}_{1}$ and $\mathcal{O}_{2}$ type of condensates. Fig. 15. In the right panel, we plot the percentage density depletion at the core as a function of the chemical potential for the two kinds of condensates. Firstly, the fact that this depletion fraction is not $100 \%$ is an indicator that we are quite far away from the solution (26) of the GP equation.

In [20], it was shown that the density depletion fraction, at zero temperature, was directly related to whether the system was BEC-like (large depletion) or BCSlike (small depletion) (for non-relativistic systems). It is interesting to note that the density depletion for holographic solitons in Fig. 15] is strongly dependent on the type of operator which condenses. The amount of density depletion for the $\mathcal{O}_{1}$ condensate is likely to be quite large near zero temperature. By contrast, $\mathcal{O}_{2}$ type condensate 


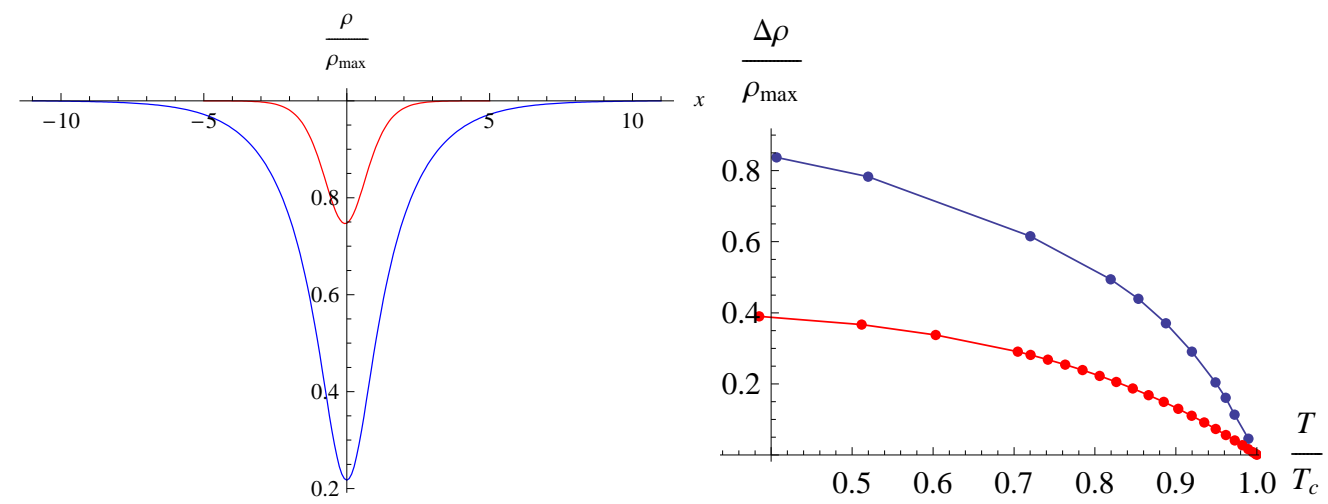

Figure 15: Left: density depletion: $\mathcal{O}_{1}$ is blue and dotted. Right: density depletion as a function of the temperature: $\mathcal{O}_{1}$ on the top and $\mathcal{O}_{2}$ on the bottom

seems to be saturating near $40 \%$ density depletion as we tend to zero temperature.

If one defines the type of superfluid (BEC or BCS) by the amount of density depletion at the core [20], then Fig. [15 suggests that the $\mathcal{O}_{1}$ system is likely to be BEC type. However, as we shall see later, it is to be emphasized that the comparison with the BCS/BEC types of superfluids is perhaps an analogy only.

\subsection{Comparison of solitons with quasiparticles}

The dark soliton we have constructed is a "macroscopic" object in this system with a characteristic length scale, namely the coherence length. On the other hand, we also have quasiparticles which are massive. These are "microscopic" excitations, and one could try to compare the inverse mass of the quasi particle with the coherence length. In a sense, since linear response is controlled by the quasiparticle mass, it will be interesting to wonder if the coherence length may be accounted for using some heuristic based on linear response theory.

The quasiparticle mass can be determined by studying the two point functions for fluctuations around the homogeneous condensate, and examining the fall-off as a function of the spatial co-ordinate $\mathrm{x}$ (we define the quasi-particle mass this way). The boundary conditions satisfied by the fluctuations in this case are "reflective" regularity conditions at the horizon. This is different from the sound mode studies in that the latter uses infalling boundary at the horizon as is appropriate for a black hole quasi-normal mode (which represents a relaxation mode for the superfluid system).

Using the AdS/CFT dictionary, the boundary quasiparticle masses arise from the poles in the bulk to boundary propagator in Euclidean AdS space [21]. Because we want the lightest masses (largest length scales), it is only necessary to solve for the lowest pole which has vanishing Matsubara frequency. Finding the lowest pole of the bulk to boundary propagator reduces to finding the values of $k^{2}$ for which one has a 
static solution to the linearized equation of motion satisfying the correct boundary conditions (see below).

In detail, we can linearize (7) to find

$$
\begin{array}{r}
f \delta R^{\prime \prime}+f^{\prime} \delta R^{\prime}-z \delta R-k^{2} \delta R+\delta R\left(\frac{A^{2}}{f}\right)+\frac{2 A \tilde{R}}{f} \delta A=0 \\
f \delta A^{\prime \prime}-k^{2} \delta A-\tilde{R}^{2} \delta A-2 A \tilde{R} \delta A=0
\end{array}
$$

where $k$ represents the (dimensionless) momentum of the particle in the $\mathrm{x}$-direction and we have dropped the tilde on the fluctuation $\delta \tilde{R}(k, z)(\tilde{R}, A$ are solutions representing the background). The presence of the interation term between $\delta \tilde{R}$ and $\delta A$ suggests that there is mixing between these two kinds of quasiparticles - very similar to the mixing between axial and vector-mesons in models of AdS/QCD [22].

The boundary conditions at the horizon are again obtained by requiring regularity of the solutions

$$
-3 \delta R^{\prime}(k, z=1)=\left(1+k^{2}\right) \delta R(k, z=1) \quad \delta A(k, z=1)=0
$$

One may understand this as a requirement that the excitation energy of the quasiparticle over the ground state energy be finite.

In order to determine the boundary conditions to be imposed at the AdS boundary, we again determine the behavior of the modes close to the boundary and require that the corresponding "non-normalizable" modes are zero. This gives

$$
\delta R(z=0)=0 \quad \delta A(z=0)=0
$$

We now solve the equations (29) subject to the above boundary condition as an eigenvalue problem for $M^{2}=-k^{2}$. For this value of $k^{2}$ there will be a vanishing eigenvalue for the linearized equations of motion, and hence a pole in the bulk to boundary propagator. The resultant graph of quasiparticle mass vs. temperature is shown in Fig. 16 along with the coherence lengths. It is interesting to note that for $\mathcal{O}_{2}$ the quasiparticle length scale tracks the soliton's condensate length scale for $\frac{\mu}{\mu_{c}}<1.2$. This is surprising in that one might have expected the lightest quasiparticle to follow the largest length scale. This might be a sign that one should also include other fields in the quasiparticle analysis (in the sense that one of the other fields has poles which track the larger length scale). To date, our numerical accuracy is insufficient to see if this is also true for $\mathcal{O}_{1}$.

\section{Discussion}

In this work, we have studied properties of one type of extended configuration allowed in the holographic superfluids described in [6]. These superfluids support dark soliton 

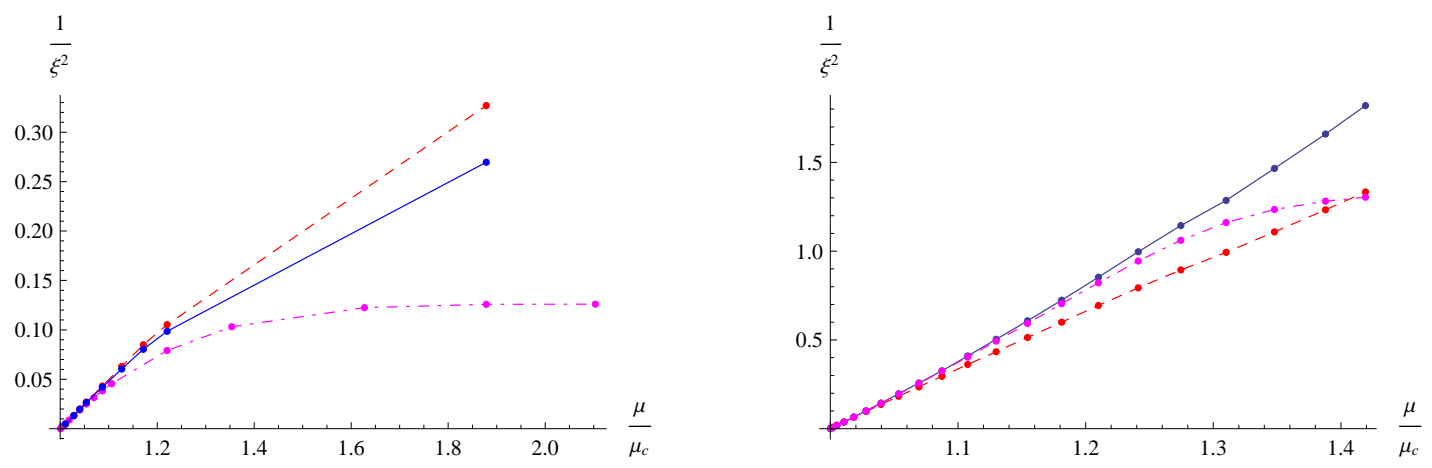

Figure 16: Quasiparticle mass (magenta, dot-dashed) as a function of $\mu$ with $\xi$ (blue) and $\xi_{q}$ (red, dashed)

: $\mathcal{O}_{1}$ on the left and $\mathcal{O}_{2}$ on the right.

solutions which are characterized by a local depletion in the charge density. This paper explored various features of these solitons as functions of the chemical potential and condensate type using numerical simulations.

Although the system we actually solved is a Maxwell-Higgs system in the presence of a black hole, the magic of the holographic AdS/CFT correspondence results in a dark soliton with parallels to the soliton of the GP equation. In fact, we found that the length scale associated with the soliton scales with the chemical potential as for soliton of the GP equation.

However, we found that the variation in the order parameter occurs with a different length scale than for the charge density. This is a feature for superfluids of both $\left\langle\mathcal{O}_{1}\right\rangle$ and $\left\langle\mathcal{O}_{2}\right\rangle$ types. The presence of these two length scales is a surprising result which is not manifest in the original gravitational system nor was it predicted by hydrodynamical studies.

A feature of these scales is that for the $\left\langle\mathcal{O}_{1}\right\rangle$ superfluid, the length scale associated with the order parameter is larger than that associated with the total charge density. As discussed in Section 4.2, this indicates an interesting spatial dependence of the non-condensate fraction of the charge density. If we use the size of order parameter as an indicator of the fraction of charge density which resides in the condensate itself, we can conclude that the non-condensate fraction of the total charge density is over dense near the dark soliton. For the $\left\langle\mathcal{O}_{2}\right\rangle$ superfluid, the relative size of the two length scales is reversed and we find that the non-condensate fraction must be under dense near the soliton core.

Spatial ordering in the components of the charge density will almost certainly affect transport phenomena near the dark soliton. The presence of different spatial orderings in the non-condensate charge density might allow one to test how the quasiparticles see the condensate differently than the non-condensate matter, although it 
should be pointed out that the relative difference in the two length scales is small for the chemical potentials discussed in this paper. This would be an interesting direction to pursue in future work.

A second feature that we found is that, at finite temperature, the density depletion in the core of the soliton is typically quite far from $100 \%$. In this sense too, the solitons of the GP equations are a bad guide. The actual value of the density depletion fraction strongly depends upon the type of holographic superfluid studied. For an $\left\langle\mathcal{O}_{1}\right\rangle$ condensate we find that the depletion fraction grows to near $100 \%$ for low temperatures, whereas for an $\left\langle\mathcal{O}_{2}\right\rangle$ condensate the depletion fraction seems to asymptote towards $40 \%$ for low temperatures.

These same gross features are known to exist in non-relativistic superfluids. In the context of the BEC-BCS crossover, it is known that BEC dark solitons have near $100 \%$ depletion fractions, while BCS superfluids have depletion fractions less than $60 \%$ [20]. In that case, the difference is associated with the characteristic size of the field which condenses. In the BEC regime it is a pointlike boson condensing while in the BCS regime the condensate is comprised of large Cooper pairs.

It should be noted that in holographic superfluids, the precise microscopic description of the system is not known. In general, it may be expected that we can construct both fermionic and bosonic quasiparticles. One could ask if an analogous classification of the size of the quasiparticles (into tightly bound BEC atoms and relatively large sized Cooper pairs) is possible in relativistic holographic superfluids. Therefore, we could hope to realize features of both types of both BEC and BCS type superfluids by tuning parameters of the gravitational description.

If we associate the depletion fraction of dark soliton with the scaling dimension of the condensing operator, it is natural to imagine that one may change the depletion fraction as one tunes the scalar mass in the gravitational action. In fact, in the works of $[16,23]$, the authors found a rich structure of ground states as the mass of the bulk scalar varied away from $L^{2} m^{2}=-2$. It would be quite interesting to explore how the properties of the quasiparticles and solitons vary with $m^{2}$ and whether one can find a crossover analogous to what is found for non-relativisitc superfluids.

Of course, one of the important question to be answered before taking the analogy to the BCS system seriously is whether there is a Fermi surface, or some aspect of a Fermi surface in the dual system. There have been several explorations in this regard and in fact, it has even been studied if one can realize a (non-)Fermi liquid holographically [24-27, 29, 30].

One potential shortcoming of our work is the fact that we do not have a smooth limit to zero temperature. This is an artefact of the probe limit [31]. Therefore, a numerically challenging problem which is potentially of much interest is to construct the fully backreacted black hole with a dark soliton. Such a solution to the gravity system would be very interesting in the sense that it would give this black hole rather novel hair. For a recent discussion along this line see [32] (of course, in string theory 
contexts, such spatially inhomogeneous hair have a complex history).

We have observed (2.2) that the presence of the condensate perturbs the gauge field background by little. Therefore, it might be interesting to explore a different pertubation expansion, by considering scalar fields in a charged black hole background. This would amount to including the backreaction of the noncondensate part, and thus correspond to an expansion in the superfluid density.

We also note that in recent works [33-35], several other extended solutions of the gravitational system were studied. In these works, non-normalizable components of some bulk fields were turned on and the solitons were then interpreted as being vortices in a superconductor. In this sense, while these authors study the same set of equations, their results are interpreted quite differently. In a subsequent work, we will present numerical results about vortices in a supefluid. The main difference with the previous work is the absence of any non-normalizable component to the bulk magnetic field.

Finally, one would like to explore such phenomena in the context of non-relativistic gauge gravity duals. In this way one might hope to model the experimentally observed dark solitons much more closely.

\section{Acknowledgements}

We would like to thank Lincoln Carr, Ari Harju and Smitha Vishveshwara for useful discussions. The numerical results presented in this work were obtained by extensive programming in Mathematica. We wish to acknowledge our debt to the makers of this software. V.K. and E.K-V. have been supported in part by the Academy of Finland grant number 1127482 .

\section{References}

[1] V. Keränen, E. Keski-Vakkuri, S. Nowling and K. P. Yogendran, "Dark Solitons in Holographic Superfluids," arXiv:0906.5217 [hep-th].

[2] J. M. Maldacena, "The large N limit of superconformal field theories and supergravity," Adv. Theor. Math. Phys. 2, 231 (1998) [Int. J. Theor. Phys. 38, 1113 (1999)] arXiv:hep-th/9711200.

[3] S. S. Gubser, I. R. Klebanov and A. M. Polyakov, "Gauge theory correlators from non-critical string theory," Phys. Lett. B 428, 105 (1998) arXiv:hep-th/9802109.

[4] E. Witten, "Anti-de Sitter space and holography," Adv. Theor. Math. Phys. 2, 253 (1998) arXiv:hep-th/9802150. 
[5] O. Aharony, S. S. Gubser, J. M. Maldacena, H. Ooguri and Y. Oz, "Large N field theories, string theory and gravity," Phys. Rept. 323, 183 (2000) arXiv:hep-th/9905111].

[6] S. A. Hartnoll, C. P. Herzog and G. T. Horowitz, "Building a Holographic Superconductor," Phys. Rev. Lett. 101, 031601 (2008) [arXiv:0803.3295 [hep-th]].

[7] S. S. Gubser, "Breaking an Abelian gauge symmetry near a black hole horizon," Phys. Rev. D 78, 065034 (2008) [arXiv:0801.2977 [hep-th]].

[8] S. A. Hartnoll, C. P. Herzog and G. T. Horowitz, "Holographic Superconductors," JHEP 0812, 015 (2008) arXiv:0810.1563 [hep-th]].

[9] C. P. Herzog, P. K. Kovtun and D. T. Son, "Holographic model of superfluidity," arXiv:0809.4870 [hep-th].

[10] E. Keski-Vakkuri and P. Kraus, "Effective theory of Quantum Hall Effect in AdS/CFT," JHEP 0809 (2008) 130 arXiv:0805.4643 [hep-th]].

[11] D. T. Son, "Toward an AdS/cold atoms correspondence: a geometric realization of the Schroedinger symmetry," Phys. Rev. D 78, 046003 (2008) arXiv:0804.3972 [hep-th]].

[12] K. Balasubramanian and J. McGreevy, "Gravity duals for non-relativistic CFTs," Phys. Rev. Lett. 101, 061601 (2008) arXiv:0804.4053 [hep-th]].

[13] I. Amado, M. Kaminski and K. Landsteiner, "Hydrodynamics of Holographic Superconductors," JHEP 0905 (2009) 021 [arXiv:0903.2209 [hep-th]].

[14] C. P. Herzog and A. Yarom, "Sound modes in holographic superfluids," arXiv:0906.4810 [hep-th].

[15] K. Maeda, M. Natsuume and T. Okamura, "Universality class of holographic superconductors," Phys. Rev. D 79 (2009) 126004 [arXiv:0904.1914 [hep-th]].

[16] O. C. Umeh, "Scanning the Parameter Space of Holographic Superconductors," JHEP 0908 (2009) 062 arXiv:0907.3136 [hep-th]].

[17] P. Basu, A. Mukherjee and H. H. Shieh, "Supercurrent: Vector Hair for an AdS Black Hole," Phys. Rev. D 79, 126004 (2009) [arXiv:0809.4494 [hep-th]].

[18] V. Balasubramanian, P. Kraus, A. E. Lawrence and S. P. Trivedi, "Holographic probes of anti-de Sitter space-times," Phys. Rev. D 59, 104021 (1999) arXiv:hep-th/9808017.

[19] I. R. Klebanov and E. Witten, "AdS/CFT correspondence and symmetry breaking," Nucl. Phys. B 556, 89 (1999) arXiv:hep-th/9905104. 
[20] M. Antezza, F. Dalfovo, L. P. Pitaevskii, and S. Stringari, "Dark solitons in a superfluid Fermi gas," Phys. Rev. A 76, 043610 (2007) arXiv:0706.0601.

[21] E. Witten, "Anti-de Sitter space, thermal phase transition, and confinement in gauge theories," Adv. Theor. Math. Phys. 2, 505 (1998) arXiv:hep-th/9803131].

[22] S. K. Domokos and J. A. Harvey, Phys. Rev. Lett. 99, 141602 (2007) arXiv:0704.1604 [hep-ph]].

[23] Y. Kim, Y. Ko and S. J. Sin, "Density driven symmetry breaking and Butterfly effect in holographic superconductors," arXiv:0904.4567 [hep-th].

[24] S. S. Lee, "A Non-Fermi Liquid from a Charged Black Hole: A Critical Fermi Ball," Phys. Rev. D 79, 086006 (2009) [arXiv:0809.3402 [hep-th]],

[25] H. Liu, J. McGreevy and D. Vegh, "Non-Fermi liquids from holography," arXiv:0903.2477 [hep-th],

[26] T. Faulkner, H. Liu, J. McGreevy and D. Vegh, "Emergent quantum criticality, Fermi surfaces, and AdS2," arXiv:0907.2694 [hep-th],

[27] M. Cubrovic, J. Zaanen and K. Schalm, "Fermions and the AdS/CFT correspondence: quantum phase transitions and the emergent Fermi-liquid," arXiv:0904.1993 [hep-th].

[28] S. J. Sin and I. Zahed, arXiv:0907.1434 [hep-th].

[29] T. Albash and C. V. Johnson, arXiv:0907.5406 [hep-th].

[30] P. Basu, J. He, A. Mukherjee and H. H. Shieh, arXiv:0908.1436 [hep-th].

[31] G. T. Horowitz and M. M. Roberts, arXiv:0908.3677 [hep-th].

[32] S. Nakamura, H. Ooguri and C. S. Park, "Gravity Dual of Spatially Modulated Phase," arXiv:0911.0679 [hep-th].

[33] T. Albash and C. V. Johnson, "Vortex and Droplet Engineering in Holographic Superconductors," arXiv:0906.1795 [hep-th].

[34] M. Montull, A. Pomarol and P. J. Silva, "The Holographic Superconductor Vortex," arXiv:0906.2396 [hep-th].

[35] K. Maeda, M. Natsuume and T. Okamura, "Vortex lattice for a holographic superconductor," arXiv:0910.4475 [hep-th]. 